\title{
The status quo and influence of self-management behaviors in convalescent stroke patients ${ }^{\dagger}$
}

Feng-Guang Guana, Mei Wang ${ }^{\mathrm{b}, *}$, Xiao-Qin Lianª

aNursing Department, The Second Affiliated People's Hospital, Fujian University of Traditional Chinese Medicine, Fuzhou, Fujian 350003, China

${ }^{b}$ School of Nursing, Fujian University of Traditional Chinese Medicine, Fuzhou, Fujian 350122, China

Received: 7 September 2017; Accepted: 8 November 2017; Published: 20 June 2018

Abstract: 0bjective: The aims of this study were to investigate the status quo of self-management behaviors in stroke patients at the recovery stage and to explore its influencing factors.

Methods: A total of 440 hospitalized convalescent stroke patients were recruited and investigated using the Basic Situation Questionnaire, Self-management Behavior Scale of Stroke, Stroke Prevention Knowledge Questionnaire and Social Support Rating Scale.

Results: The mean self-management behavior score was $(151.95 \pm 23.58)$, and dimensions in descending order were as follows: dietary management, drug safety management, social function and interpersonal relationships, life management, emotion management, rehabilitation exercise management and disease management. Five regional self-management behavior scores were statistically significant, and the scores from Minnan and Minzhong of the Fujian province, China, were higher than the others. Gender, age, family income and self-management behavior were significantly correlated $(P<0.05)$; educational level, stroke knowledge level, social support level and self-management behavior were positively correlated, and the difference was statistically significant $(P<0.01)$. Conclusions: The overall self-management level of convalescent stroke patients should be improved to strengthen health education; focus on the educational level, which is relatively low; strengthen the social support system of patients; stimulate the enthusiasm and initiative of self-management disease patients to promote disease rehabilitation and improve the quality of life.

Keywords: stroke • self-management $\bullet$ behavior $\bullet$ root cause analysis

(c) Shanxi Medical Periodical Press.

\section{Introduction}

Stroke is the second leading cause of death in the world, according to the survey of the American Heart Association in $2015 .{ }^{1}$ In the analysis of the Chinese stroke prevention and control report (2015), stroke is the leading cause of death in China, and its economic burden is as high as 40 billion yuan per year. ${ }^{2}$ The high morbidity, high fatality rate, high disability rate, high recurrence rate, complications and long course of the disease

\footnotetext{
† This project was supported by 2016 Fujian Provincial Science and Technology Department of the Pilot Project (No. 2016Y0047).
}

seriously reduce the quality of life for stroke patients and put great pressure on the family and society. ${ }^{3}$ The rehabilitation of stroke patients is multifaceted, among which rehabilitation management is an extremely important link. ${ }^{4}$ Self-management is the monitoring and management of disease through proactive health behaviors, which reduce the effects of disease through treatment, disease maintenance and health promotion. ${ }^{5}$ Studies ${ }^{6-8}$ have shown that the self-management ability and quality of life of stroke patients are positively related and that good self-management behavior is essential for patients in stroke recovery to maximize the recovery of bodily functions, promote healthy behaviors, reduce recurrence and improve long-term survival. The purposes 
of this study were to investigate the self-management behaviors of stroke recovery patients through a questionnaire survey and to analyze their influencing factors to provide references and ideas for building self-management skills in convalescent stroke patients.

\section{Objects and methods}

\subsection{Study subjects}

A total of 450 stroke recovery patients from nine hospitals of the Fujian province were selected. The inclusion criteria were cerebrovascular disease diagnosis of the fourth diagnostic criteria by brain computed tomography (CT) or magnetic resonance imaging (MRI) in stroke patients; the period of stable disease, with "restore" being the guiding principle of clinical research on new drugs of traditional Chinese medicine treatment for stroke in the recovery period, 15 days -6 months after the onset of stroke; clear consciousness; ability to use text or language communication; Barthel Index (BI) score $>20$; absence of swallowing dysfunction; and the ability to participate voluntarily. The exclusion criteria were the mental scale assessment for dementia or mental illness; association with other serious diseases, such as heart, kidney and liver insufficiency; and the complete loss of activity ability. The sample size of this study was eight times the number of items in the survey tool, "stroke self-management behavior scale", considering a $10 \%$ falling rate, with the final sample of 450 cases.

\subsection{Research tools}

\subsubsection{The basic situation questionnaire}

According to our research purpose and the stroke characteristics of patients, a total of 14 items were included: gender, age, educational level, marital status, occupation, family income, medical payment, accompanying relatives and friends, visit type, frequency, treatment time, hospitalization time, complications, disease knowledge acquisition approaches and evaluation of the rehabilitative effect of others.

\subsubsection{Self-management behavior scale of stroke patients}

The self-management behavior scale of stroke patients, developed by $W^{2} a^{9}$ in 2009, is used to reflect the change in self-management behaviors in stroke patients and consists of seven dimensions and 51 items, with good reliability and validity; Cronbach's $\alpha$ coefficient was 0.835 . This scale comprised specific content of disease management (11 items), drug safety management (five items), catering management (nine items), daily life management (eight items), emotion management (five items), social function and interpersonal management (six items) and rehabilitation management (11 items). With the 5-point Likert scoring method, the total score was 50-250, and the higher the score, the better the self-management behavior of patients.

\subsubsection{Stroke prevention knowledge questionnaire}

The stroke prevention knowledge questionnaire was designed by Zhang ${ }^{10}$ and covers daily life, exercise, diet, medication, blood pressure monitoring, risk factors, stroke symptoms and stroke treatment in eight dimensions, for a total of 36 items. In this scale, Cronbach's $\alpha$ coefficient was 0.812 and there was good reliability and validity. Each entry option was "know" or "do not know", with know=1 and do not know=0; the higher the score, the higher the level of stroke knowledge.

\subsubsection{Social Support Rating Scale (SSRS)}

The SSRS ${ }^{11}$ consists of three dimensions composed of 10 items, including objective support (three items), subjective support (four items) and the degree of social support (three items). Four or more scores were used, and the sum of the 10 items was the total score of social support. In items 1-4 and 8-10, each patient made only one selection from $A$ to $D$, scoring 1-4 points; for the fifth question, each patient selected from A to $E$, for a total score of 5 points. Each item was 1-4 points from "no" to "full support"; for the sixth and seventh questions, if the answer was "no source", then the score was 0 point, and then, the following source was answered by selecting the source number. The higher the score, the higher the level of social support received by participants.

\subsection{Survey methodology}

A questionnaire was distributed after a unified training study to meet the inclusion criteria for stroke recovery inpatients and to obtain informed consent. The patient was told how to fill out the survey, and if in doubt, a unified survey language was used to explain it. The patient himself or herself filled out the questionnaire on the spot, and if the patient could not fill it out alone, the researcher assisted the patient and avoided introducing words during this period. After being completed, the questionnaire was recovered on the spot to ensure the integrity of the data. A total of 450 questionnaires were issued and recovered, among which 440 were valid; thus, the effective participation rate was $97.78 \%$. 


\subsection{Statistical methods}

Data collection was conducted with an Excel database, using IBM SPSS Statistics 20.0 statistical software for the descriptive statistics of general demographic data; data were counted as percentage (\%), data were measured as mean and standard deviation (descriptive) and comparison between groups were carried out using a $t$-test and single factor analysis of variance $(P<0.05)$. The knowledge score for stroke prevention and treatment was graded according to score: $>80 \%$ as good, $60 \%-80 \%$ as medium and $<60 \%$ as not good. Pearson correlation analysis and multiple linear regression analysis were used to evaluate self-management in stroke patients and recovery factors; $P<0.01$ was considered as statistically significant.

\section{Results}

\subsection{Self-management level of patients with stroke convalescence}

The self-management behavior score in the stroke recovery stage ranged from 75 to 211 , with a mean of $151.95 \pm 23.58$. The seven latitudes were sorted according to the scoring index from high to low: dietary management, safe medication management, social functioning and interpersonal relationships, daily life management, emotion management, rehabilitation exercise management and disease management. For the Fujian province, the five regions of the selfmanagement of patients with the stroke recovery score were statistically significant $(P=0.000, P<0.05)$; there were significant differences in the four latitudes: disease management, medication management, food safety management and rehabilitation management. See Tables 1 and 2 for details.

\subsection{Single-factor analysis of self- management and demographic data with stroke convalescence}

There was a significant effect $(P<0.05)$ found for stroke recovery score of self-management behavior differences in the single-factor analysis of self-management level by age, gender, educational level and family income per month; no statistically significant differences were found

\begin{tabular}{|c|c|c|c|c|c|c|}
\hline Outcome & $\begin{array}{l}\text { Scoring } \\
\text { range }\end{array}$ & Minimum & Maximum & $\begin{array}{l}\text { Actual score } \\
\quad(\mathrm{M} \pm \mathrm{SD})\end{array}$ & $\begin{array}{c}\text { Scoring } \\
\text { index (\%) }\end{array}$ & $\begin{array}{c}\text { Descending } \\
\text { order }\end{array}$ \\
\hline Dietary management & $8-40$ & 15 & 40 & $28.63 \pm 5.25$ & 71.58 & 1 \\
\hline Safe medication management & $5-25$ & 5 & 25 & $16.66 \pm 4.52$ & 66.64 & 2 \\
\hline Social functioning and interpersonal management & $6-30$ & 6 & 30 & $19.68 \pm 4.86$ & 65.60 & 3 \\
\hline Daily life management & $8-40$ & 8 & 38 & $25.92 \pm 5.45$ & 64.80 & 4 \\
\hline Emotion management & $5-25$ & 5 & 23 & $16.15 \pm 3.80$ & 64.40 & 5 \\
\hline Rehabilitation exercise management & $7-35$ & 7 & 35 & $22.22 \pm 7.25$ & 63.49 & 6 \\
\hline Disease management & $11-55$ & 11 & 54 & $22.68 \pm 8.00$ & 41.24 & 7 \\
\hline Total score & $50-250$ & 75 & 211 & $151.95 \pm 23.58$ & - & - \\
\hline
\end{tabular}

Table 1. Scores of self-management dimensions in the stroke recovery stage $(n=440)$.

\begin{tabular}{|c|c|c|c|c|c|c|c|}
\hline Item & $\begin{array}{c}\text { Minbei } \\
(M \pm S D)\end{array}$ & $\begin{array}{l}\text { Mindong } \\
(\mathrm{M} \pm \mathrm{SD})\end{array}$ & $\begin{array}{l}\text { Minnan } \\
(\mathrm{M} \pm \mathrm{SD})\end{array}$ & $\begin{array}{c}\text { Minxi } \\
(\mathrm{M} \pm \mathrm{SD})\end{array}$ & $\begin{array}{l}\text { Minzhong } \\
(\mathrm{M} \pm \mathrm{SD})\end{array}$ & $F$ & $P$ \\
\hline Self-management score & $156.92 \pm 23.26$ & $162.12 \pm 29.55$ & $168.28 \pm 27.55$ & $149.94 \pm 26.25$ & $166.59 \pm 22.42$ & 5.435 & 0.000 \\
\hline Disease management & $21.75 \pm 7.45$ & $21.79 \pm 8.32$ & $24.30 \pm 9.67$ & $19.16 \pm 6.42$ & $25.23 \pm 8.44$ & 4.910 & 0.001 \\
\hline Safe medication management & $16.18 \pm 4.54$ & $15.79 \pm 5.08$ & $17.26 \pm 4.83$ & $14.68 \pm 5.54$ & $17.23 \pm 4.06$ & 3.018 & 0.018 \\
\hline Dietary management & $27.81 \pm 4.40$ & $29.26 . \pm 6.02$ & $29.74 \pm 5.49$ & $26.82 \pm 4.77$ & $29.25 \pm 4.85$ & 3.684 & 0.006 \\
\hline Daily life management & $25.58 \pm 5.28$ & $25.86 \pm 5.98$ & $26.39 \pm 5.41$ & $24.10 \pm 5.01$ & $25.82 \pm 5.60$ & 1.492 & 0.204 \\
\hline Emotion management & $16.14 \pm 3.51$ & $16.28 \pm 3.95$ & $16.10 \pm 3.43$ & $16.16 \pm 4.46$ & $15.93 \pm 4.13$ & 0.057 & 0.994 \\
\hline $\begin{array}{l}\text { Social functioning and interpersonal } \\
\text { management }\end{array}$ & $20.31 \pm 4.84$ & $18.19 \pm 4.86$ & $19.83 \pm 4.70$ & $20.36 \pm 4.73$ & $18.93 \pm 4.69$ & 2.080 & 0.083 \\
\hline Rehabilitation exercise management & $29.15 \pm 9.50$ & $34.95 \pm 8.50$ & $34.66 \pm 8.87$ & $28.66 \pm 11.17$ & $34.20 \pm 8.66$ & 7.413 & 0.000 \\
\hline
\end{tabular}

Table 2. Self-management behavior scores of Mindong, Minxi, Minnan, Minbei, and Minzhong, Fujian province ( $n=440)$ 


\begin{tabular}{|c|c|c|c|c|c|}
\hline Characteristics & $n$ & $\%$ & $\begin{array}{l}\text { Self-management behavior } \\
\text { score }(M \pm S D)\end{array}$ & $t / F$ & $P<0.05$ \\
\hline \multicolumn{6}{|l|}{ Gender } \\
\hline Male & 252 & 57.3 & $149.89 \pm 23.92$ & 4.531 & 0.034 \\
\hline Female & 188 & 42.7 & $154.71 \pm 22.90$ & & \\
\hline \multicolumn{6}{|l|}{ Age (years) } \\
\hline$<40$ & 14 & 3.2 & $155.64 \pm 25.92$ & 3.938 & 0.009 \\
\hline $40-49$ & 56 & 12.7 & $142.20 \pm 24.82$ & & \\
\hline $50-59$ & 71 & 16.1 & $151.55 \pm 21.61$ & & \\
\hline$\geq 60$ & 299 & 68.0 & $153.70 \pm 23.34$ & & \\
\hline \multicolumn{6}{|l|}{ Educational level } \\
\hline Primary school & 153 & 34.8 & $124.09 \pm 15.19$ & 439.981 & 0.000 \\
\hline Junior middle school & 107 & 24.3 & $143.60 \pm 7.19$ & & \\
\hline High school & 103 & 23.4 & $155.65 \pm 6.51$ & & \\
\hline College degree or above & 77 & 17.5 & $177.40 \pm 13.87$ & & \\
\hline \multicolumn{6}{|l|}{ Marital status } \\
\hline Not married & 14 & 3.2 & $144.07 \pm 24.40$ & 0.498 & 0.737 \\
\hline Married & 372 & 84.5 & $152.47 \pm 24.01$ & & \\
\hline Divorce & 10 & 2.3 & $149.70 \pm 22.37$ & & \\
\hline Separation & 2 & 0.5 & $149.50 \pm 24.75$ & & \\
\hline Widowed & 12 & 9.5 & $150.57 \pm 19.97$ & & \\
\hline \multicolumn{6}{|c|}{ Monthly income per household (yuan) } \\
\hline$<1000$ & 42 & 9.5 & $149.95 \pm 21.25$ & 2.641 & 0.049 \\
\hline 1000-1999 & 74 & 16.8 & $146.82 \pm 24.95$ & & \\
\hline 2000-2999 & 142 & 32.3 & $150.90 \pm 23.78$ & & \\
\hline$\geq 3000$ & 182 & 41.4 & $155.36 \pm 23.04$ & & \\
\hline \multicolumn{6}{|l|}{ Medical payment mode } \\
\hline Public expense & 25 & 5.7 & $146.04 \pm 24.79$ & 2.650 & 0.072 \\
\hline Medical insurance & 307 & 69.8 & $153.61 \pm 23.53$ & & \\
\hline At their own expense & 108 & 24.5 & $148.60 \pm 23.10$ & & \\
\hline \multicolumn{6}{|l|}{ Caregiver type } \\
\hline Relatives & 334 & 75.9 & $150.93 \pm 24.50$ & 1.560 & 0.369 \\
\hline Professional workers & 75 & 17.1 & $155.96 \pm 20.07$ & & \\
\hline Nanny & 9 & 2.0 & $163.22 \pm 22.07$ & & \\
\hline Other & 5 & 1.1 & $158.60 \pm 25.99$ & & \\
\hline None & 17 & 3.9 & $146.35 \pm 16.62$ & & \\
\hline \multicolumn{6}{|c|}{ Frequency of visiting relatives and friends } \\
\hline Every day & 246 & 55.9 & $152.00 \pm 24.33$ & 1.053 & 0.369 \\
\hline 2-6 times a week & 125 & 28.4 & $152.42 \pm 21.57$ & & \\
\hline 1 time a week & 43 & 9.8 & $154.72 \pm 21.60$ & & \\
\hline Less than 1 time a week & 26 & 5.9 & $144.62 \pm 28.39$ & & \\
\hline
\end{tabular}

Table 3. Single-factor analysis of demographic data of patients in the stroke recovery stage $(n=440)$. 
for the scores of other projects $(P>0.05)$. The scores are presented in Table 3.

\subsection{Stroke knowledge level}

The score for prevention and control knowledge of stroke recovery patients was $(26.80 \pm 7.78)$, and the overall score of patients was medium, among which stroke treatment and risk factors were the lowest. The scores from diet, daily life, exercise, blood pressure monitoring, stroke symptoms, medication, risk factors, and stroke treatment followed from high to low. See Table 4 for details.

\subsection{Correlation analysis of self-management behaviors and stroke knowledge level, social support level and education level}

Because the variables were continuous and continued to have a normal distribution, Pearson's correlation analysis was performed The results showed that the level of stroke knowledge, social support and educational level were positively related to self-management

\begin{tabular}{lccc}
\hline Outcome & $\begin{array}{c}\text { Actual score } \\
(\mathrm{M} \pm \mathrm{SD})\end{array}$ & $\begin{array}{c}\text { Scoring } \\
\text { index }(\%)\end{array}$ & Classification \\
\hline \hline Diet & $3.38 \pm 0.98$ & 84.50 & Good \\
Daily life & $6.67 \pm 1.73$ & 83.38 & Good \\
Motion & $3.14 \pm 1.11$ & 78.50 & Medium \\
Blood pressure monitoring & $1.39 \pm 0.77$ & 69.50 & Medium \\
Signs of stroke & $4.15 \pm 2.13$ & 69.17 & Medium \\
Medication & $3.45 \pm 1.54$ & 69.00 & Medium \\
Risk factors & $3.34 \pm 1.77$ & 66.84 & Medium \\
Stroke management & $1.28 \pm 0.83$ & 64.00 & Medium \\
Total score & $26.80 \pm 7.78$ & 74.44 & Medium \\
\hline
\end{tabular}

Table 4. Stroke prevention and treatment knowledge score in the stroke recovery stage $(n=440)$. behaviors, and the correlation coefficients were $r=0.252$, $r=0.193$ and $r=0.863$, respectively. The difference was statistically significant, $P<0.01$.

\subsection{Multiple regression analysis on influencing factors of self-management behaviors in the stroke recovery stage}

According to the results of the single-factor analysis, we selected educational level, stroke knowledge level, and social support level as independent variables and selfmanagement score as the dependent variable for use in the multiple linear stepwise regression analysis. The collinearity diagnostic tolerance of the regression equation was $0.953-0.976(>0.1)$, and the variance inflation factor (VIF) was 1.024-1.049 $(<10)$. It can be considered that there was no multicollinearity between the three independent variables, which accords with basic multiple linear regression assumptions. The results show that the education level, stroke knowledge level, and social support level are the factors that influence the self-management behaviors of stroke patients in recovery. Three of the total load contributions of size are as follows: educational level, social support level, stroke knowledge level, and a common interpretation of self-management behavior level. The variance is $75.8 \%$ (Table 5).

\section{Discussion}

\subsection{Status quo of self-management behavior in the stroke recovery stage}

In this group of patients in stroke recovery, the total mean score of patient self-management was $151.95 \pm 23.58$, higher than that of $\mathrm{Mu}$ et al., ${ }^{12}$ and this group is included in the study on the recovery of stroke patients, self-cognition and behavior for knowledge management attention; this score is higher than in the group of first-stroke patients. Self-management has

\begin{tabular}{|c|c|c|c|c|c|}
\hline Variables & $\begin{array}{c}\text { Partial regression } \\
\text { coefficient }\end{array}$ & Standard error & $\begin{array}{c}\text { Standard regression } \\
\text { coefficient }\end{array}$ & $T$ & $P<0.01$ \\
\hline Constant & 91.983 & 3.429 & - & 26.827 & 0.000 \\
\hline Educational level & 16.823 & 0.484 & 0.837 & 34.773 & 0.000 \\
\hline Stroke knowledge level & 0.277 & 0.073 & 0.091 & 3.811 & 0.000 \\
\hline Social support level & 0.449 & 0.148 & 0.072 & 3.026 & 0.003 \\
\hline
\end{tabular}

Table 5. Influencing factors of self-management in the stroke recovery stage $(n=440)$. Note: $F=458.631, P=0.000(<0.001)$, coefficient of decision $R^{2}=0.759$ and adjusted $R^{2}=0.758$. 
seven dimensions according to the score, from high to low: dietary management, drug safety management, social functioning and interpersonal relationships, life management, emotion management, and rehabilitation of disease management. The highest scores for patients were for dietary management and drug safety management, because the subjects of this study was in the period of hospitalization, health care provider continue to provide health education to patients with disease, and are in compliance during hospitalization regarding good medication and diet, which influence, to a certain extent, the two latitude scores. Patients with poor performance in rehabilitation exercise management and disease management behavior had the worst disease management behavior, which is consistent with the studies of Wang et al. ${ }^{13}$ and Liu et al. ${ }^{14}$ who analyzed a number of factors that may be related to disease rehabilitation in the long term: lack of perseverance, imperfect rehabilitation equipment, lack of knowledge and skills for rehabilitation, medical expenses, etc.; the authors suggest strengthening the rehabilitation training of patients with guidance and helping patients build self-confidence with disease monitoring.

The self-management behavior scores for the five regions of the Fujian province were statistically significant, and there were significant differences in disease management, safe medication management, dietary management and rehabilitation exercise management. The two regional scores for Minnan and Minzhong were higher than the others, and the analysts believed that this outcome was related to economic integration, as these areas represent the political, economic, cultural and scientific research centers of the Fujian province.

\subsection{Analysis of the influencing factors of self-management behaviors in the stroke recovery stage}

\subsubsection{Educational level}

Educational level is an important predictor of self-management behaviors of patients, since the higher the level of education, the higher the level of health selfmanagement, and these results were similar to those of Mu et al. ${ }^{12}$ and Li et al. ${ }^{15}$ Patients with a high educational level learn, understand and absorb knowledge and information more comprehensively and deeply and can obtain self-health care knowledge in more ways; they have better communication with medical staff; are more willing to adopt self-beneficial treatment, a rehabilitation plan and care and engage in more conscious and active learning and the implementation of effective disease self-management behaviors. Therefore, for patients, the medical staff should follow the principles of the person and take a path of culturally relevant health education. For patients with a low degree of culture, the medical staff should use understandable words and talk about, explain, and repeat the key disease self-management points many times. For highly educated patients, it is necessary to meet self-actualization (Tables 2 and 4).

\subsubsection{Social support level}

Social support can effectively help patients mobilize psychological resources to cope with negative emotions and meet their needs through the process of hardship and suffering together by providing material support and psychological support and by helping patients adapt to success. The findings of this study confirm that social support levels can influence self-management behaviors, which is consistent with the findings of previous studies. ${ }^{16,17}$ Good social support can effectively promote physical and mental development in patients and improve self-management behavior. In clinical nursing, we should fully mobilize the social resources of patients, especially focusing on the good role of family function, and ensure the quality of social support.

\subsubsection{Stroke knowledge level}

This study shows that stroke knowledge level is an important factor in self-management behavior and that the cognitive level of stroke management and risk factors is low. Most patients do not know the prime window of opportunity for stroke treatment, often missing the best time to treat. Therefore, for these patients in stroke recovery, knowledge about the disease should be emphasized during self-management health education, especially the treatment and risk factors of stroke.

\section{Conclusions}

The overall level of the self-management of stroke patients needs to be improved and should be strengthened through health education by paying attention to lower cultural degree, by helping to mobilize patient social support systems and overall, by stimulating the enthusiasm and initiative of self-management disease patients to promote disease recovery and improve quality of life.

\section{Conflicts of interest}

All contributing authors declare no conflicts of interest. 


\section{References}

1. Mozaffarian D, Benjamin EJ, Go AS, et al. Heart disease and stroke statistics - 2015 update: A report from the American Heart Association. Circulation. 2015;131:e29-322.

2. National Stroke Prevention Committee of national health and Family Planning Commission. Chinese stroke prevention and control report (2015). 2015 (in Chinese).

3. Sivan M, Gallagher J, Makower S, et al. Homebased Computer Assisted Arm Rehabilitation (hCAAR) robotic device for upper limb exercise after stroke: Results of a feasibility study in home setting. J Neuroeng Rehabil. 2014;11:1.

4. Yang WW, Zhang J, Tong L. Research progress of self-management measuring tool for stroke. Chin Nurs Manag. 2017;17:410-414 (in Chinese).

5. Jiang YL, Wang YJ, Feng Y, Wang Y, Tang Q, Wang $R$. Construction and evaluation of self-management items in patients with cerebral apoplexy at convalescence stage. J Nurs Sci. 2014;29:4-7 (in Chinese).

6. Gao $\mathrm{CH}$, Huang XL, Huang J, Zhang W, Wang W, Cai $\mathrm{JH}$. Effect of self-management on improving activities of daily living and quality of life of stroke patients in community. Chin J Rehabil Theory Pract. 2014;20:789-793 (in Chinese).

7. Zhao $\mathrm{YY}$, Zhang $\mathrm{AH}$. Correlation between selfmanagement behaviors and anxiety, depression and quality of life in patients with coronary heart disease undergoing interventional therapy. J Nurs Sci. 2016;31:8-11 (in Chinese).

8. Satink T, Josephsson S, Zajec J, Cup EH, de Swart BJ, Nijhuis-van der Sanden MW. Self-management develops through doing of everyday activities-a longitudinal qualitative study of stroke survivors during two years post-stroke. BMC Neurol. 2016;16:221.
9. Wang YJ. Construction and Application of SelfManagement Project for Stroke. Chengdu: Chengdu University of TCM; 2009 (in Chinese).

10. Zhang XP. Correlation between Health Behavior and Quality of Life in Patients with Brain Stroke after Discharge. Guangzhou: Sun Yat-sen University; 2009 (in Chinese).

11. Xiao SY. The theoretical basis and research application of social support rating scale. J Clin Psych. 1994;4:98-100 (in Chinese).

12. Mu X, Li J, Liu RJ, Zheng XY. Study on self-management and influencing factors of first stroke patients. Chin J Nurs. 2016;51:289-293 (in Chinese).

13. Wang $Y J$, Jiang $Y L$, Wang $Y$, Feng $Y$, Wang $R$, Tang $Q$. Investigation on knowledge level and selfmanagement behavior of stroke patients at recovery stage. Chin Nurs Res. 2013;27:3090-3092 (in Chinese).

14. Liu $Y$, Jing $Y$, Zhao $Y$. Analysis of health behaviors and influencing factors in young patients with stroke. Chin J Nurs. 2012;47:58-61 (in Chinese).

15. Li J, Sun PF, Xia HG, Cheng CX. Status quo and influencing factors of health self-management among elderly people with stroke in Hebei. Chin $J$ Public Health. 2015;31:140-143 (in Chinese).

16. Shangliu WX, Shen MF, Chen WY, Basanti P, Zhu $D Q$. Investigation of symptom management selfefficacy and its influencing factors in patients with chronic heart failure. Chin J Nurs. 2014;49:674-678 (in Chinese).

17. Tian $X H$, Feng $Y R$, Chen $C X$. The influence of family, community and social support on general self-efficacy of stroke elderly. Chin Nur Res. 2016;30:2532-2534 (in Chinese). 